\title{
On the problem of non-smoothness of non-reflexive second conjugate spaces
}

\section{Ivan Singer}

\begin{abstract}
We prove that if $E$ is a Banach space which has a subspace $G$ such that the conjugate space $G^{*}$ contains a proper norm closed linear subspace $V$ of characteristic 1 , then $E^{* *}$ is not smooth and there exist in $\pi_{E}(E)$ points of non-smoothness for $E^{* *}$, where $\pi_{E}: E \rightarrow E^{* *}$ is the canonical embedding. We show that the spaces $E$ having such a subspace $G$ constitute a large proper subfamily of the family of all non-reflexive Banach spaces.
\end{abstract}

1 .

A Banach space $E$ is called smooth if for every $x \in E$ with $\|x\|=1$ there exists a unique $f \in E^{*}$ with $\|f\|=1$ such that $f(x)=1$. If $E$ is not smooth, any $x \in E$ with $\|x\|=1$ for which there are $f_{1}, f_{2} \in E^{*}$ with $f_{1} \neq f_{2},\left\|f_{1}\right\|=\left\|f_{2}\right\|=1$, satisfying $f_{1}(x)=f_{2}(x)=1$, is called a point of non-smoothness for $E$.

Giles [6], Day ([3], p. 70), Pełczyński, Phelps and Rainwater [12] have proved that a non-reflexive Banach space $E$ has non-smooth third conjugate space $E^{* * *}$ and that for any $f \in E^{*}$ with $\|f\|=1$ wich does

Received 29 January 1975. This paper was prepared during the author's visit, from May to September 1974, to the Département d'Informatique, Université de Montréal, on leave from the Institute of Mathematics of the Romanian Academy of Sciences. The author thanks Professor J.H.M. Whitfield for calling his attention to papers [12] and [6] and for stimulating discussions; and $\mathrm{Dr} J . R$. Giles for valuable comments. 
not attain its norm on the unit sphere of $E, \pi_{E^{*}}(f)$ is a point of nonsmoothness for $E^{* *}$, where $\pi_{E^{*}}: E^{*} \rightarrow E^{* * *}$ is the canonical embedding. In the present paper we shall study the problem whether the second conjugate $E^{* *}$ of a non-reflexive Banach space $E$ is non-smooth, raised by Rainwater [12], and the problem whether one can find points of nonsmoothness for $E^{* *}$ already in $\pi_{E}(E)$, where $\pi_{E}: E \rightarrow E^{* *}$ is the canonical embedding. In $\$ 2$ we shall prove that this is indeed the case for a large family of non-reflexive spaces $E$, including non-reflexive conjugate spaces (which yields again the result mentioned above) and spaces $E$ for which dens $E<$ dens $E^{*}$, where dens $E$ denotes the density character of $E$ (that is, the smallest cardinality of a dense set in $E$ ). In $\$ 3$ we shall prove that there exist non-reflexive Banach spaces $E$ which do not satisfy our sufficient condition for the non-smoothness of $E^{* *}$.

The notations and terminology used here will be the standard ones (see, for example, [3], [13]). We recall (see [4]) that the characteristic of a subspace $V$ (by subspace we shall always mean norm closed linear subspace) of a conjugate space $E^{*}$ is the number

$$
r(V)=\inf _{\substack{x \in E \\ x \neq 0}} \sup _{\substack{f \in V \\ f \in \leq 1}}\left|f\left(\frac{x}{\|x\|}\right)\right|=\inf _{\substack{x \in E,\|x\|=1 \\ \Phi \in V^{2}}}\left\|\pi_{E}(x)+\Phi\right\|,
$$

where $V^{\mathcal{L}}=\left\{\Phi \in E^{* *} \mid \Phi(f)=0(f \in V)\right\}$. Thus, $r\left(E^{*}\right)=1$, $0 \leq r(V) \leq 1 \quad\left(V \subset E^{*}\right)$, and we have $r(V)=1$ if and only if

$$
\|x\|=\sup _{\substack{f \in V \\\|f\|=1}}|f(x)|,(x \in E),
$$

or equivalently, if and only if the projection $p$ of $\pi_{E}(E) \oplus V^{\perp}$ onto $\pi_{E}(E)$ along $V^{\perp}$ has norm $\|p\|=I$. Also, we recall that if the conjugate space $E^{*}$ is separable, the norm of $E$ is called [5] a Kadec'Klee norm if the relations $\left\{f_{n}\right\} \subset E^{*}, f \in E^{*}, f_{n} \stackrel{w^{*}}{\longrightarrow} f,\left\|f_{n}\right\| \rightarrow\|f\|$ imply $\left\|f_{n}-f\right\| \rightarrow 0$. In [5], Corollary 1, it was proved that every Banach space $E$ with separable conjugate space $E^{*}$ admits an equivalent norm such that in this new norm for every proper subspace $V$ of $E^{*}$ we have 
$r(V)<1$, namely, any equivalent Kadec'-Klee norm has this property (equivalent Kadec'-Klee norms exist by [8], [9]).

2 .

DEFINITION 1. We shall say that a Banach space $E$ has

(a) property $\left(\mathrm{CH}_{1}\right)$, if the conjugate space $E^{*}$ contains a proper subspace $V$ (that is, such that $V \neq E^{*}$ ) of characteristic $r(V)=1$,

(b) property $\left(\mathrm{SCH}_{1}\right)$, if $E$ contains a subspace $G$ with property $\left(\mathrm{CH}_{1}\right)$.

THEOREM 1. If $E$ is a Banach space with property $\left(\mathrm{SCH}_{1}\right)$, then $E^{* *}$ is not smooth. Moreover, there exist in $\pi_{E}(E)$ points of nonsmoothness for $E^{* *}$.

Proof. Let us first assume that the theorem holds for every Banach space with property $\left(\mathrm{CH}_{l}\right)$ and let $G$ be a subspace, with property $\left(C B_{1}\right)$, of $E$. Then, by our assumption, $G^{* *} \equiv G^{+*} \subset E^{* *}$ is not smooth, hence $E^{* *}$ is not smooth. Also, by our assumption, let $x \in G$ be such that $\pi_{G}(x)$ is a point of non-smoothness for $G^{* *}$, so there exișt $\psi_{1}, \psi_{2} \in G^{* * *}$ with $\psi_{1} \neq \psi_{2}$ such that $\left\|\psi_{1}\right\|=\left\|\psi_{2}\right\|=1$, $\psi_{1}\left(\pi_{G}(x)\right)=\psi_{2}\left(\pi_{G}(x)\right)=1$. If $u$ is the identical embedding of $G$ into $E$, then $\pi_{E^{\mu}}=u^{* *} \pi_{G}, u^{* * *}$ maps $E^{* * *}$ onto $G^{* * *}$ and for each $\psi \in G^{* * *}$ there exists $\Xi \in E^{* * *}$ with $u^{* * *}(\Xi)=\psi,\|\Xi\|=\|\psi\|$. Hence, if $\Xi_{1}, \Xi_{2} \in E^{* * *}$ are such that $u^{* * *}\left(\Xi_{j}\right)=\psi_{j},\left\|\Xi_{j}\right\|=\left\|\psi_{j}\right\|=1$ $(j=1,2)$, then $\Xi_{1} \neq \Xi_{2}$ and $\Xi_{j}\left(\pi_{E} u(x)\right)=\Xi_{j}\left(u^{* *} \pi_{G}(x)\right)=u^{* * *}\left(\Xi_{j}\right)\left(\pi_{G}(x)\right)=\psi_{j}\left(\pi_{G}(x)\right)=1, \quad(j=1,2)$, so $\pi_{E} \mu(x)$ is a point of non-smoothness for $E^{* *}$. Thus, it is enough to prove Theorem 1 under the assumption that $E$ has property $\left(\mathrm{CH}_{1}\right)$.

Let $V$ be a proper subspace of $E^{*}$ with $r(V)=1$. It will be 
enough to prove that there exist in $\pi_{E}(E)$ points of non-smoothness for the subspace $\pi_{E}(E) \oplus V^{+}$of $E^{* *}$, since then by the Hahn-Banach Theorem these will be points of non-smoothness also for $E^{* *}$.

Since $V \neq E^{*}$ and $V$ is norm-closed, there exists $f \in E^{*} \backslash V$ with $\|f\|=1$ such that $f(x)=1$ for some $x \in E$ with $\|x\|=1$ (indeed, by the Bishop-Phelps Theorem [1] those $f \in E^{*}$ with $\|f\|=1$ which admit such an $x$ are norm dense in the unit sphere of $E^{*}$ ). We shall show that $\pi_{E}(x) \in \pi_{E}(E)$ is a point of non-smoothness for $\pi_{E}(E) \oplus V^{2}$.

Let us denote by $\rho$ the restriction mapping

$$
\rho(\Xi)=\left.\Xi\right|_{\pi_{E}(E) \oplus V^{+}},\left(\Xi \in E^{* * *}\right),
$$

and by $p$ the projection of $\pi_{E}(E) \oplus V^{\perp}$ onto $\pi_{E}(E)$ along $V^{\perp}$ (hence, by $r(V)=1$ we have $\|p\|=1$ ). We shall show that the functionals

$$
\varphi_{1}=\rho \pi_{E^{*}}(f), \quad \varphi_{2}=\left(\pi_{E}^{-1} p\right)^{*}(f) \in\left(\pi_{E}(E) \oplus V^{+}\right)^{*}
$$

satisfy, for any $\Phi \in V^{\perp}$ with $\Phi \neq 0$ (such a $\Phi$ exists, as $f \in E^{*} \backslash V$ ),

$$
\varphi_{1}(\Phi) \neq 0=\varphi_{2}(\Phi), \quad \varphi_{1}\left(\pi_{E}(x)\right)=\varphi_{2}\left(\pi_{E}(x)\right)=1,\left\|\varphi_{1}\right\|,\left\|\varphi_{2}\right\| \leq 1,
$$

which will complete the proof. Indeed, we have

$$
\begin{aligned}
& \varphi_{1}(\Phi)=\rho \pi_{E^{*}}(f)(\Phi)=\left.\pi_{E^{*}}(f)\right|_{\pi_{E}(E) \oplus V^{+}}(\Phi)=\pi_{E^{*}}(f)(\Phi)=\Phi(f) \neq 0, \\
& \varphi_{2}(\Phi)=\left(\pi_{E}^{-1} p\right)^{*}(f)(\Phi)=f\left(\pi_{E}^{-1} p(\Phi)\right)=f\left(\pi_{E}^{-1}(0)\right)=f(0)=0, \\
& \varphi_{1}\left(\pi_{E^{\prime}}(x)\right)=\left.\pi_{E^{*}}(f)\right|_{\pi_{E}(E) \oplus V^{\perp}}\left(\pi_{E}(x)\right)=\pi_{E^{*}}(f)\left(\pi_{E}(x)\right)=\pi_{E^{\prime}}(x)(f)=f(x)=1 \text {, } \\
& \varphi_{2}\left(\pi_{E}(x)\right)=f\left(\pi_{E}^{-1} p\left(\pi_{E}(x)\right)\right]=f\left(\pi_{E}^{-1} \pi_{E}(x)\right)=f(x)=\perp \text {, } \\
& \left\|\varphi_{1}\right\| \leq\|\rho\|\left\|\pi_{E^{*}}\right\|\|f\|=1, \quad\left\|\varphi_{2}\right\| \leq\left\|p^{*}\right\|\left\|\left(\pi_{E}^{-1}\right)^{*}\right\|\|f\|=1,
\end{aligned}
$$

which completes the proof of Theorem 1 .

REMARK 1. Clearly, for every $\pi_{E}(x) \in \pi_{E}(E)$ with $\left\|\pi_{E}(x)\right\|=1$, the 
unit ball $S_{E^{* *}}$ admits a weak* closed support hyperplane at $\pi_{E}(x)$; that is, of the form $H_{1}=\left\{\Psi \in E^{* *} \mid \pi_{E^{*}}(f)(\Psi)=1\right\}$ for some $f \in E^{*}$ with $\|f\|=1$. The above proof shows that if $E$ is smooth and has property $\left(\mathrm{CH}_{1}\right)$, then there exists a $\pi_{E}(x) \in \pi_{E}(E)$ with $\left\|\pi_{E}(x)\right\|=1$, such that $S_{E^{* *}}$ admits also a weak* dense support hyperplane at $\pi_{E}(x)$; that is, of the form $H_{2}=\left\{\Psi \in E^{* *} \mid \Xi(\Psi)=1\right\}$, for some $\Xi \in E^{* * *} \backslash \pi_{E^{*}}\left(E^{*}\right)$ with $\|\Xi\|=1$ (containing the support line through $\pi_{E}(x)$ and $\pi_{E}(x)+\Phi$, where $\Phi \in V^{\perp}$ is as above).

REMARK 2. The conclusion of Theorem 1 remains valid, with a similar proof, for any space $E$ for which there exists a triple $(\Phi, f, x)$ with $\Phi \in E^{* *}, f \in E^{*}, x \in E$ of norm $\|\Phi\|=\|f\|=\|x\|=1$, such that $\Phi(f) \neq 0, f(x)=1,\left\|\pi_{E}(x)+\alpha \Phi\right\| \geq\left\|\pi_{E}(x)\right\|=1$ for all scalars $\alpha$.

Indeed, it is enough to replace then $\pi_{E}(E) \oplus V^{\perp}$ by the twodimensional subspace $\left[\pi_{E}(x)\right] \oplus[\Phi]$ of $E^{* *}$ and the functionals $\varphi_{1}, \varphi_{2}$ above by

$$
\varphi_{1}^{\prime}=\rho_{\left[\pi_{E}(x)\right] \oplus[\Phi]^{\pi_{E}}}(f), \varphi_{2}^{\prime}=p_{0}^{*} \rho_{\left[\pi_{E}(x)\right]}\left(\pi_{E}^{-1}\right)^{*}(f) \in\left(\left[\pi_{E}(x)\right] \oplus[\Phi]\right)^{*},
$$

where $\left.\rho\right|_{\Gamma}(\Xi)=\left.\Xi\right|_{\Gamma}$ for all $\Xi \epsilon E^{* * *}$ and for any subspace $\Gamma$ of $E^{* *}$ and where $p_{0}$ denotes the projection of $\left[\pi_{E}(x)\right]+[\Phi]$ onto $\left[\pi_{E}(x)\right]$ along $[\Phi]$.

REMARK 3. There may exist also other points of non-smoothness for $E^{* *}$. For example, if there exists a $\Phi \in E^{* *} \backslash \pi_{E}(E)$ with $\|\Phi\|=1=\operatorname{dist}\left(\Phi, \pi_{E}(E)\right)$ which attains its norm at some $f \in E^{*}$ with $\|f\|=1$, then $\Phi$ is a point of non-smoothness for $E^{* *}$. Indeed, on the one hand, $\pi_{E^{*}}(f)(\Phi)=1$, so the weak* closed hyperplane $H_{1}=\left\{\Psi \in E^{* *} \mid \pi_{E^{*}}(f)(\Psi)=1\right\}$ supports $S_{E^{* *}}$ at $\Phi$ and, on the other hand, by a corollary of the Hahn-Banach Theorem there exists $\Xi \in \pi_{E^{(}}(E)^{\perp} \subset E^{* * * \backslash \pi_{E^{*}}\left(E^{*}\right)}$ with $\|\equiv\|=1, \Xi(\Phi)=1$, so the weak ${ }^{*}$ dense 
hyperplane $H_{2}=\left\{\Psi \in E^{* *} \mid E(\Psi)=1\right\}$ also supports $S_{E^{* *}}$ at $\Phi$. However, it is well known that there are Banach spaces for which there exists no $\Phi \in E^{* *} \backslash \pi_{E}(E)$ such that $\|\Phi\|=1=\operatorname{dist}\left(\Phi, \pi_{E}(E)\right)$.

Let us give now some corollaries of Theorem 1 .

COROLLARY 1. If dens $E<$ dens $E^{*}$, then $E^{* *}$ is not smooth and there exist in $\pi_{E}(E)$ points of non-smoothness of $E^{* *}$.

Proof. It is well known that $E$ has property $\left(\mathrm{CH}_{1}\right)$ (it is enough to take a dense set $\left\{x_{i}\right\}_{i \in I}$ in $S_{E}$ with card $I=$ dens $E$ and then for each $i \in I$ a functional $f_{i} \in S_{E^{*}}$ with $f_{i}\left(x_{i}\right)=1$ and to put $V=\left[f_{i}\right]_{i \in I}$, the closed linear span of $\left.\left\{f_{i}\right\}_{i \in I}\right\}$.

We also obtain as a corollary the following slight improvement of the Giles-Rainwater result:

COROLLARY 2. If $E$ contains a subspace $G$ isometric to a nonreflexive conjugate space $B^{*}$, then $E^{* *}$ is not smooth and there exist in $\pi_{E}(E)$ points of non-smoothness for $E^{* *}$.

Proof. It is well known that $G$ has property $\left(\mathrm{CH}_{1}\right)$ (take $V \subset G^{*}$ to be the image of $\pi_{B}(B) \subset B^{* *}$ under the isometry $B^{* *} \equiv G^{*}$ ).

Finally, let us observe that in the above cases $E$ cannot have any one of the properties implied by the smoothness of $E^{* *}$, for example:

COROLLARY 3. If $E$ satisfies the condition of Theorem I lor of Corolzary 1 or 2), then $E^{* * *}$ is not strictly convex.

Let us also observe that if a Banach space $E$ contains a subspace $G$ isomorphic to $c_{0}$, then $E^{* *}$ is not omooth. Indeed, $G^{* *} \equiv G^{L 1} \subset E^{* *}$ is then isomorphic to $l^{\infty}$ and hence is not smooth, by a result of Day [2], so $E^{* *}$ is not smooth.

If there exists a non-reflexive space $E_{0}$ with smooth second conjugate space $E_{0}^{* *}$, then (by passing to a subspace, if necessary) we may assume that $E_{0}$ is separable (even that $E_{0}$ has a basis, by [10]) and 
then by the above results, $E_{0}$ must have the following properties:

(a) $E_{0}^{*}$ is separable;

(b) $E_{0}$ contains no subspace isomorphic to $c_{0}$;

(c) $E_{0}$ contains no non-reflexive subspace isometric to a conjugate Banach space $B^{*}$.

Therefore it is natural to raise

PROBLEM 1. Does the quasi-reflexive space $E=J$ of James [7] admit an equivalent norm $\|\cdot\| \|$ such that $\left(E^{* *},\|\| \cdot \|\right)$ is smooth?

3 .

Clearly, a Banach space with property $\left(\mathrm{SCH}_{1}\right)$ is non-reflexive. Unfortunately, there are non-reflexive spaces which do not have property $\left(\mathrm{SCH}_{1}\right)$, as we shall show below (and therefore Theorem 1 alone does not imply that every non-reflexive Banach space $E$ has non-smooth second confugate space).

THEOREM 2. Let $E$ be a non-reflexive Banach space with separable conjugate space $E^{*}$. Then $E$ admits an equivalent norm $\|\cdot\| \|$ such that $(E, \quad\|l \cdot\|)$ does not have property $\left(\mathrm{SCH}_{1}\right)$. In fact, every equivalent Kadec'-Klee norm on $E$ satisfies this condition.

Proof. By $\S 1$, for any equivalent Kadec'-Klee norm $\|\cdot\| \|$, $(E,\|\cdot \cdot\|)$ does not have property $\left(\mathrm{CH}_{1}\right)$. Therefore it will be sufficient to prove that for any subspace $G \subset E$ the restriction to $G$ of a Kadec'-Klee norm $\|\cdot\|$ on $E$ is a Kadec'-Klee norm on $G$.

Let $\left\{\varphi_{n}\right\} \subset G^{*}, \varphi \in G^{*}, \varphi_{n} \stackrel{\omega^{*}}{\longrightarrow} \varphi,\left\|\varphi_{n}\right\| \rightarrow\|\varphi\|$. We shaIl show that every subsequence $\left\{\varphi_{n_{k}}\right\} \subset\left\{\varphi_{n}\right\}$ contains a subsequence $\left\{\varphi_{n_{k}}\right\}$ such that $\left\|\varphi_{n_{k_{m}}}-\varphi\right\| \rightarrow 0$, which will complete the proof (since then $\left.\left\|\varphi_{n}-\varphi^{i}\right\| \rightarrow 0\right)$. 
Let $\left\{f_{n}\right\} \subset E^{*}, f \in E^{*}$ be any Hahn-Banach extensions (that is, with the same norm) of $\left\{\varphi_{n}\right\}$ and $\varphi$ respectively. Then

$$
\sup _{k}\left\|f_{n_{k}}\right\|=\sup _{k}\left\|\varphi_{n_{k}}\right\|<\infty \text {, }
$$

whence, since $E$ is separable, $\left\{f_{n_{k}}\right\}$ contains a subsequence $\left\{f_{n_{k_{m}}}\right\}$ such that $f_{n_{k_{m}}} \stackrel{w^{*}}{\longrightarrow} h \in E^{*}$. Then, from $f_{\left.n_{k_{m}}\right|_{G}}=\varphi_{n_{k_{m}}} \stackrel{w^{*}}{\longrightarrow} \varphi$, we obtain $\left.h\right|_{G}=\varphi$, whence $\|\varphi\| \leq\|h\|$. On the other hand,

$$
\|h\| \leq \frac{1 i m}{m+\infty}\left\|f_{n_{k_{m}}}\right\|=\frac{1 i m}{m^{+\infty}}\left\|\varphi_{n_{k_{m}}}\right\|=\|\varphi\|,
$$

hence $\left\|f_{n_{k_{m}}}\right\| \rightarrow\|h\|$. Consequently, since the norm on $E$ is a Kadec'Klee norm, $\left\|f_{n_{k_{m}}}-\hbar\right\| \rightarrow 0$, whence, by restriction to $G$, $\left\|\varphi_{n_{k_{m}}-\varphi}\right\| \| \rightarrow$, which completes the proof of Theorem 2.

REMARK 4. Theorem 2 shows, in particular, that there exist nonreflexive Banach spaces $E$ in which there is no asymptotically monotone non-shrinking basic sequence (although it is well known that in every Banach space $E$ there are asymptotically monotone basic sequences and in every non-reflexive space $E$ there are non-shrinking basic sequences); indeed, for an asymptotically monotone basic sequence the coefficient functionals span a proper subspace of characteristic 1 . In [11] it was proved that for every non-reflexive Banach space $E$, there exists in $E^{* *} \backslash \pi_{E}(E)$ an asymptotically monotone non-shrinking basic sequence.

REMARK 5. After this paper was completed, $\mathrm{Dr}$ J.R. Giles communicated to us that Corollary 1 can be also proved by using a result of Tacon, [14], Lemma 6, p. 420. 


\section{References}

[1] Errett Bishop and R.R. Phelps, "The support functionals of a convex set", Convexity, 27-35 (Proc. Sympos. Pure Maths 7. Amer. Math. Soc., Providence, Rhode Island, 1963).

[2] Mahlon M. Day, "Strict convexity and smoothness of normed spaces", Trans. Amer. Math. Soc. 78 (1955), 516-528.

[3] Mahlon M. Day, Normed Zinear spaces, 3rd ed. (Ergebnisse der Mathematik und ihrer Grenzgebiete, 21. Springer-Verlag, Berlin, Heidelberg, New York, 1973).

[4] J. Dixmier, "Sur un théorème de Banach", Duke Math. J. 15 (1948), 1057-1071.

[5] Dick van Dulst and I van Singer, "On Kadec-Klee norms on Banach spaces", Studia Math. (to appear).

[6] J.R. Giles, "A non-reflexive Banach space has non-smooth third conjugate space", Canad. Math. Bul2. 17 (1974), 117-119.

[1] Robert C. James, "A non-reflexive Banach space isometric with its second conjugate space", Proc. Nat. Acad. Sci. USA 37 (1951), $174-177$.

[8] М.Н. Надець [M.Y. Kadec'], "Про зө'язон МІн слабою та снлъною збІнністн" [On the connection between weak and strong convergence], Dopovidi Akad. Nauk Ukraïn. RSR 1959, 949-952.

[9] V. Klee, "Mappings into normed linear spaces", Fund. Math. 49 (1960-1961), 25-34.

[10] A. Pełczyński, "A note on the paper of I. Singer 'Basic sequences and reflexivity of Banach spaces'", Studia Math. 21 (1961-1962), $371-374$.

[11] A. Pełczyński, "A proof of Eberlein-S̆mulian theorem by an application of basic sequences", Bulz. Acad. Polon. Sci. Ser. Sci. Math. Astronom. Phys. $12(1964), 543-548$.

[12] John Rainwater, "A non-reflexive Banach space has non-smooth third conjugate space", preprint. 
[13] Ivan Singer, Bases in Banach spaces I (Die Grundlehren der mathematischen Wissenschaften, 154. Springer-Verlag, Berlin, Heidelberg, New York, 1970).

[14] D.G. Tacon, "The conjugate of a smooth Banach space", Bulz. Austral. Math. Soc. 2 (1970), 415-425.

Département d'Informatique,

Université de Montréal,

Canada;

Institute of Mathematics,

Calea Grivitei 21 ,

Bucuresti,

Romania. 